University of Wollongong

Research Online

Faculty of Engineering - Papers (Archive)

Faculty of Engineering and Information

Sciences

May 1994

\title{
Plasmon assisted resonant tunneling in a double barrier heterostructure
}

C. Zhang

University of Wollongong, czhang@uow.edu.au

M. L. Lerch

University of Wollongong, mlerch@uow.edu.au

A. D. Martin

University of Wollongong

Philip E. Simmonds

University of Wollongong, simmonds@uow.edu.au

L. Eaves

University of Nottingham, UK

Follow this and additional works at: https://ro.uow.edu.au/engpapers

Part of the Engineering Commons

https://ro.uow.edu.au/engpapers/279

\section{Recommended Citation}

Zhang, C.; Lerch, M. L.; Martin, A. D.; Simmonds, Philip E.; and Eaves, L.: Plasmon assisted resonant tunneling in a double barrier heterostructure 1994.

https://ro.uow.edu.au/engpapers/279

Research Online is the open access institutional repository for the University of Wollongong. For further information contact the UOW Library: research-pubs@uow.edu.au 


\title{
Plasmon Assisted Resonant Tunneling in a Double Barrier Heterostructure
}

\author{
C. Zhang, ${ }^{1}$ M. L. F. Lerch, ${ }^{1}$ A. D. Martin, ${ }^{1}$ P. E. Simmonds, ${ }^{1}$ and L. Eaves ${ }^{2}$ \\ ${ }^{1}$ Department of Physics, University of Wollongong, New South Wales 2522, Australia \\ ${ }^{2}$ Department of Physics, University of Nottingham, Nottingham, NG7 2RD, England
}

(Received 17 January 1994)

\begin{abstract}
When a double barrier semiconductor structure is biased near a tunneling resonance, charge can accumulate in the quantum well. Coupling between this two dimensional electron gas and the tunneling current is investigated. Experimental data taken inside a region of apparent bistability in one device reveal a satellite on the high energy side of the current resonance in the $I(V)$ characteristic. A theoretical model based on the many-body transfer Hamiltonian formalism shows that a plasmon excitation has a remarkably similar structure. Magnetic field data support the plasmon satellite interpretation.
\end{abstract}

PACS numbers: $72.10 . \mathrm{Di}, 71.45 . \mathrm{Gm}, 73.40 . \mathrm{Kp}$

Semiconductor double barrier structures (DBSs) have undergone considerable development since the seminal work of Tsu and Esaki [1]. Of particular relevance to the work reported here is the prediction and observation of negative differential resistance (NDR) [2] and intrinsic apparent bistability [3] in the electrical characteristics $[I(V)$ curves $]$ of DBSs. Apparent bistability can arise when charge buildup in the central quantum well raises the resonant level in the well (due to the electron-electron interaction) and the resulting potential drop across the collector barrier tips the bell shaped resonance curve over to the right, giving a Z-shaped (tristable) characteristic and is particularly pronounced in devices with asymmetric barriers. Such a characteristic gives the appearance of bistability because the central arm of the $Z$ is inaccessible to conventional measurement techniques. Although the appearance of bistability can be caused by extrinsic effects [4], intrinsic bistability has been realized in appropriately designed structures $[3,5]$. An $I(V)$ curve

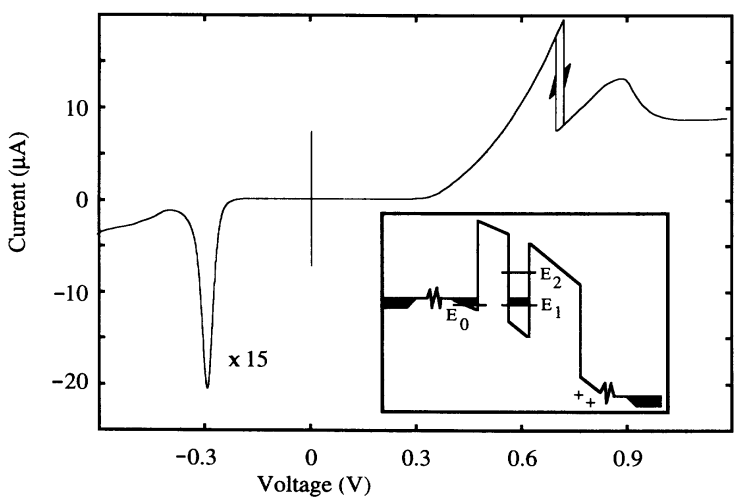

FIG. 1. $I(V)$ characteristic of the first tunneling resonance in both bias directions for the asymmetric DBS at $2 \mathrm{~K}$. Measurements were made using conventional techniques and the arrows indicate where current switching is observed thus delineating the region of apparent bistability. The broad peak at $\approx 0.9 \mathrm{~V}$ is due to LO-phonon assisted tunneling. Inset: Schematic conduction band diagram for the device under forward bias. measured conventionally for an asymmetric DBS at $2 \mathrm{~K}$ is shown in Fig. 1. Note, in reverse bias, where charge buildup is insignificant, NDR rather than bistability is observed, providing strong support for the intrinsic nature of the forward bias bistability. There is also no evidence of any structure within the NDR region of the reverse bias resonance.

It is well known that electron-LO phonon interactions can give rise to resonant satellites (see Fig. 1) [6]. In this Letter we present a previously unreported satellite which we identify as an interaction between tunneling electrons and the two dimensional electron gas (2DEG) in the quantum well. Its observation is made possible by a recently developed experimental technique [7] which employs an active load line with a positive slope (corresponding to a negative series resistance) to probe the region of apparent bistability where the satellite is concealed. Figure 2 shows the interior of the bistability delineated in Fig. 1. The satellite appears as a broad structure on the side of the main resonance. Although the satellite is at a lower voltage than the main resonance

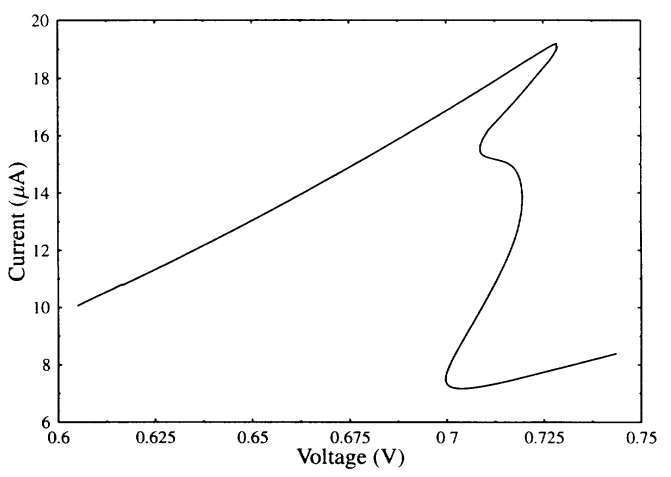

FIG. 2. Probing the region of apparent bistability with a positively sloping load line reveals the satellite on the high energy side of the main resonance. Outside this region the $I(V)$ curve is equivalent to that measured conventionally. The theory together with other experimental data presented in this paper indicate that the satellite peak may be associated with plasmon assisted tunneling. 
peak in the $I(V)$ curve it actually lies on the high energy side when the effect of the electron-electron interaction is removed (see theory). It develops as the device is cooled below $20 \mathrm{~K}[8]$ and is distinct from the LO-phonon replica. We propose a theoretical model for the satellite based on coupling between the tunneling current and the quantum well plasmon (QWP) excitation in the well near resonance. In the following we shall show that the calculated current within such a model exhibits remarkably similar structure to the experimental result. Suppression of the satellite when a magnetic field is applied parallel to the tunneling current is consistent with the identification of this structure as a plasmon assisted tunneling (PAT) resonance.

The double barrier structure described here has already been characterized in detail [9]. The active region of the device consists of a $5.8 \mathrm{~nm}$ quantum well sandwiched between $\mathrm{Al}_{0.4} \mathrm{Ga}_{0.6} \mathrm{As}$ barriers of widths 8.3 and $11.1 \mathrm{~nm}$. Lightly doped GaAs spacer layers separate this region from the heavily doped $n^{+}$contact regions. An applied bias voltage causes an accumulation layer and associated 2DEG to form adjacent to the emitter barrier. At the peak of the first resonance magnetocapacitance and luminescence studies [9] indicate a charge density in the well of $\approx 2 \times 10^{11}$ electrons $\mathrm{cm}^{-2}$.

The theoretical model employed here to treat the electron-QWP coupling is very similar to those used in the problems of electron-phonon coupling [10-12] and deep-electron x-ray emission [13]. The effect of electronQWP coupling on resonant tunneling has not, to the best of our knowledge, been discussed although it is expected to be much stronger than the electron-phonon coupling. We have solved a resonant tunneling model including electron-QWP coupling for the case where a significant electron charge density exists in the resonant well. Here the QWP is expressed in terms of an equivalent boson field and electron-boson coupling is treated in the same way as in electron-phonon coupling. We calculate the total energy-dependent transmission probability $T(\epsilon)$, for an electron with energy $\epsilon$ incident from the emitter onto the resonant level where it interacts with the QWP and is then transmitted to the collector. Because of the electron-plasmon coupling, the resulting $T(\epsilon)$ exhibits a shoulder or a weak peak in the high bias side of the main resonance peak, representing the collective excitation assisted resonant tunneling.

From an energy and momentum conservation point of view, the in-plane density excitations can indeed have a significant effect on the transport properties perpendicular to the plane. Let the total energy $\epsilon=\epsilon_{k}+\epsilon_{z}$ where $\epsilon_{k}=\hbar^{2} k^{2} / 2 m$ ( $\mathbf{k}$ is the momentum along the plane) and $\epsilon_{z}$ is the energy perpendicular to the plane. The plasmon energy is $\hbar \omega_{q}=\hbar \sqrt{2 \pi e^{2} n q / m}$. To emit such a plasmon, all $q$ has to be supplied by the momentum transfer along the plane $q=\left|\mathbf{k}-\mathbf{k}^{\prime}\right|$. However, the energy transfer corresponding to this momentum transfer is only a fraction of the plasmon energy. $\epsilon_{\mathbf{k}}-\epsilon_{\mathbf{k}-\mathbf{q}} \approx \hbar^{2} \mathbf{k} \cdot \mathbf{q} / m=\left(\hbar^{2} k q / m\right) \cos \theta$. Since $k \leq k_{f}$, $\left(\hbar^{2} k q / m\right) \cos \theta$ is smaller than $\xi=\hbar^{2} q k_{f} / m$. Here $\xi$ is the boundary of single particle excitation near the resonance $\left(k_{f} \approx k_{f}^{c}\right)$, where $k_{f}\left(k_{f}^{c}\right)$ is the Fermi wave vector in the emitter (well). Therefore a significant change in $\epsilon_{z}$ is required especially for $k=0$ states in the emitter (left) lead and the tunneling current should exhibit the effect of electron-QWP coupling. Let us recall that in the electron-LO phonon coupling case, one can usually neglect the parallel (to the plane) momentum exchange and assume that the phonon energy is solely supplied by $\epsilon_{z}-\epsilon_{z}^{\prime}$ due to its large value and the weak dispersion at zero $q$ (we should mention that recent work by Zou and Chao [11] which includes the parallel momentum does improve the peak-to-valley ratio for LO-phonon scattering). Here for the case of electron-QWP coupling, one must retain explicitly the $k$ dependence of the total tunneling rate.

Our model Hamiltonian consists of three terms, $H=$ $H_{e}+H_{p}+H_{I}$, where $H_{e}$ is the Hamiltonian for the electrons including the elastic coupling to the tunneling barriers,

$$
\begin{aligned}
H_{e}= & \sum_{p, \nu, \mathbf{k}}\left(\epsilon_{p, \nu}+\epsilon_{\mathbf{k}}\right) a_{p, \nu, \mathbf{k}}^{\dagger} a_{p, \nu, \mathbf{k}}+\sum_{\mathbf{k}}\left(\epsilon_{c}+\epsilon_{\mathbf{k}}\right) a_{c, \mathbf{k}}^{\dagger} a_{c, \mathbf{k}} \\
& +\sum_{p, \nu, \mathbf{k}}\left[V_{p, \nu} a_{c, \mathbf{k}}^{\dagger} a_{p, \nu, \mathbf{k}}+\text { c.c. }\right] .
\end{aligned}
$$

Here $\nu=l(r)$ refers to the emitting (collecting) lead with $\epsilon_{z}(\nu)=\epsilon_{p, \nu}, p$ is the momentum component perpendicular to the interface, and $c$ refers to the center well with quasibound energy level $\epsilon_{c}$. The tunneling matrix element $V_{p, \nu}$ between the leads and the quantum well is calculated according to Bardeen's prescription [14] with a one dimensional potential which includes both the band discontinuities and the applied bias. The term $H_{p}$ is a bosonlike field Hamiltonian representing the QWP,

$$
H_{p}=\sum_{\mathbf{q}} \hbar \omega_{q} b_{\mathbf{q}}^{\dagger} b_{\mathbf{q}}
$$

where $\omega_{q}$ is the QWP frequency and only its long wavelength limit will be used below. The electron-plasmon coupling $H_{I}$ can be written as

$$
H_{I}=\sum_{\mathbf{k}, \mathbf{q}} g_{q}\left(b_{\mathbf{q}}^{\dagger}+b_{\mathbf{q}}\right) a_{c, \mathbf{k}+\mathbf{q}}^{\dagger} a_{c, \mathbf{k}}
$$

which only allows momentum transfer along the plane. In the above expression, we use $g_{q}$ to denote the electronplasmon coupling strength. This coupling strength can be obtained by writing down the electron self-energy due to electron-electron interaction in the plasmon pole approximation, then comparing the result with the selfenergy due to electron-phonon interaction [13]. For electron QWP coupling, $\left|g_{q}\right|^{2}=n v_{q} \omega_{q}$ where $v_{q}$ is the 2D Fourier component of Coulomb potential and $n$ is the 2D carrier density in the quantum well.

By applying the $S$-matrix scattering theory, the total probability $T_{t}\left(\epsilon_{z}, \mathbf{k}\right)$ for an electron with energy $\left(\epsilon_{k}, \epsilon_{z}\right)$ 
in the emitter to be transmitted to the collector can be written as a product of the elastic coupling to the two leads and Fourier transform of a Green's function from the resonant level in the well [10,11],

$$
T_{t}\left(\epsilon_{z}, \mathbf{k}\right)=\Gamma_{l} \Gamma_{r} \int_{0}^{\infty} d s \int_{0}^{\infty} d t e^{i \epsilon_{z}(t-s)} \sum_{\mathbf{k}^{\prime}} e^{i \epsilon_{\mathbf{k}^{\prime}}(t-s)} \theta(t) \theta(s)\left\langle a_{c, \mathbf{k}}(t-s) a_{c, \mathbf{k}^{\prime}}^{\dagger}(t) a_{c, \mathbf{k}^{\prime}}(t) a_{c, \mathbf{k}}^{\dagger}(0)\right\rangle
$$

Here $\Gamma_{l(r)}$ is the decay rate of the resonant level due to the elastic coupling to the left (right) lead, calculated within the wide band approximation [10]. In Eq. (4) the angular brackets indicate thermal average. The fouroperator correlator can be calculated using the cumulant expansion method $[11,13]$ which includes the high order vertex corrections. We obtain

$$
T_{t}\left(\epsilon_{z}, \mathbf{k}\right)=\frac{2 \Gamma_{l} \Gamma_{r}}{\Gamma} \int_{0}^{\infty} d t \exp \left[i \Omega_{\mathbf{k}} t-\frac{1}{2} \Gamma t+\sum_{\mathbf{q}} f_{\mathbf{q}}\right]
$$

where $\Gamma=\Gamma_{l}+\Gamma_{r}, \Omega_{\mathbf{k}}=\left(\epsilon_{z}-\epsilon_{c}+\lambda_{\mathbf{k}}\right)$, and

$$
\lambda_{\mathbf{k}}=\sum_{\mathbf{q}} \frac{\left|g_{q}\right|^{2}}{\omega_{q}^{2}-\Delta^{2}}\left[\omega_{q}-\left(1+2 N_{q}\right) \Delta\right] .
$$

Here the $q$ summation is restricted to $q<q_{c}=r_{s} k_{f}^{c}$, the cutoff wave number beyond which the plasmon becomes heavily damped. The plasma parameter $r_{s}=$ $e^{2} k_{f}^{c} / 2 E_{f}^{c}=m e^{2} / \hbar^{2} k_{f}^{c}$ is a measure of coupling strength in an electron gas [15]. In Eq. (6), $N_{q}=\left[\exp \left(\beta \omega_{q}\right)-1\right]^{-1}$ and $\Delta=\epsilon_{\mathbf{k}}-\epsilon_{\mathbf{k}-\mathbf{q}}$. The function $f_{q}$ in Eq. (5) is given as

$$
\begin{aligned}
f_{\mathbf{q}}= & \frac{\left|g_{q}\right|^{2}}{\left(\omega_{q}^{2}-\Delta^{2}\right)^{2}}\left[\left(\omega_{q}^{2}+\Delta^{2}\right)\left(2 N_{q}+1\right)-2 \omega_{q} \Delta\right] \\
& -\frac{\left|g_{q}\right|^{2}\left(\omega_{q}^{2}+\Delta^{2}\right)}{\left(\omega_{q}^{2}-\Delta^{2}\right)^{2}}\left[2 N_{q} \cos \left(\omega_{q} t\right)+e^{-i \omega_{q} t}\right] e^{i \Delta t} \\
& -\frac{2\left|g_{q}\right|^{2} \omega_{q} \Delta}{\left(\omega_{q}^{2}-\Delta^{2}\right)^{2}}\left[i 2 N_{q} \sin \left(\omega_{q} t\right)-e^{-i \omega_{q} t}\right] e^{-i \Delta t}
\end{aligned}
$$

For constant $\omega_{q}$, this is just the previous result for the

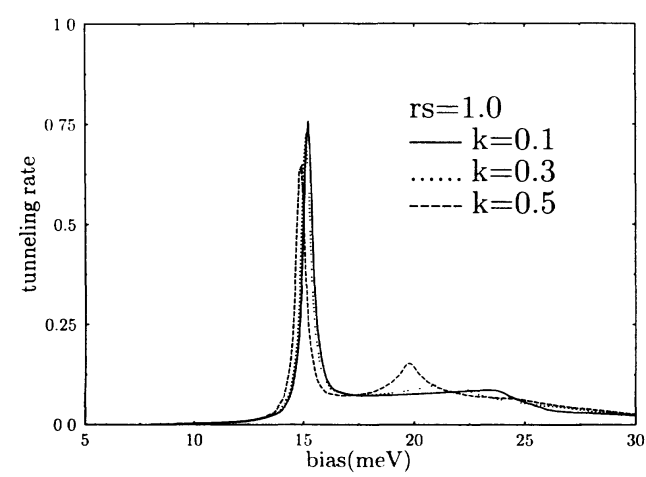

FIG. 3. Plot of tunneling rate as a function of incident energy for several different in-plane momenta expressed in terms of $k_{f}$. Note the shift of the main peak due to the QWP. case of electron-LO phonon coupling $[10,11]$. It should be mentioned here that the complex structure of $f_{q}$ and $T_{t}\left(\epsilon_{z}, \mathbf{k}\right)$ does not alter the sum rule for the total tunneling rate. Integrating over $\epsilon_{z}$ introducing a factor $\delta(t)$ ensures the sum rule because $f_{\mathbf{q}}(t=0)=0$, regardless of the $k$ value.

In Fig. 3, we present the result of $T_{t}\left(\epsilon_{z}, \mathbf{k}\right)$ for several different values of $k$ at $T=0 \mathrm{~K}$. Here (also in Fig. 4) the bias ( $x$ axis) is related to $\epsilon_{z}$ through $\epsilon_{c}-\epsilon_{z}=$ bias which in turn is proportional to the total applied bias and the $\epsilon_{c}$ is chosen to be $20 \mathrm{meV}$. It can be clearly seen that there exists a PAT satellite at a bias slightly higher (several $\mathrm{meV}$ ) than the main peak. Since the plasmon energy is $q$ dependent, the distance between the main peak and the side peak only represents the integral effect of all $\omega_{q}$ from 0 to $q_{c}$. However, this distance must reduce as $k$ increases. At $k=0$, after emitting a plasmon, the electron must gain momentum $q$ and energy $\epsilon_{q}$. This energy gain in the $x-y$ plane and the plasmon energy must be compensated by an energy loss in the $z$ direction, $\epsilon_{z}-\epsilon_{z}^{\prime}=\epsilon_{q}+\hbar \omega_{q}$. Therefore the distance between the peaks is the largest for $k=0$. As $k$ increases, the re-

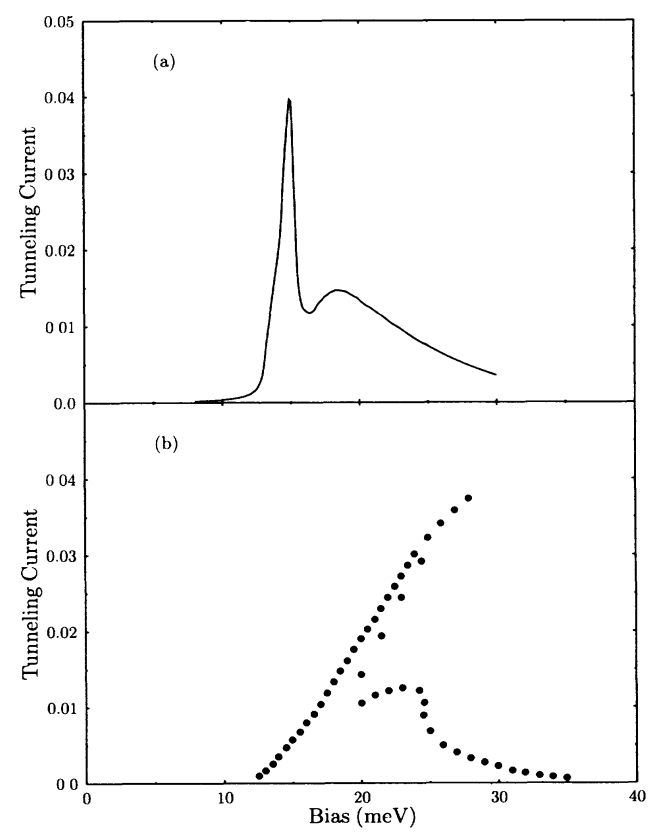

FIG. 4. (a) Plot of tunneling current as a function of applied bias; the current is expressed in units of ene $\left(2 E_{e} / m\right)^{1 / 2}$, where $n_{e}$ is the charge density in the emitter and $E_{e}$ is the energy level of the emitter. (b) Same as (a) but with the electron feedback effect in the resonant well. 


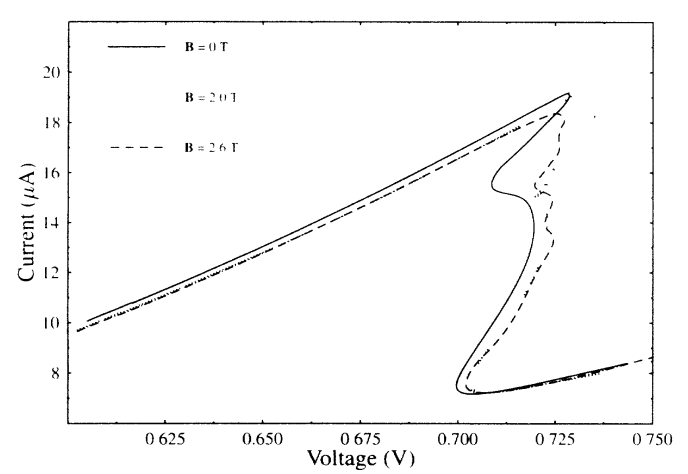

FIG. 5. Effect of a magnetic field $(\mathbf{B} \| \mathbf{J})$ on the PAT satellite peak. Once the Landau levels are fully resolved the satellite peak is no longer observed.

quired energy change in $\epsilon_{z}$ gets smaller and the satellite peak approaches the main peak. In the meantime the peak becomes lower and broader due to increasing phase breaking scattering processes and the peak-to-valley ratio becomes smaller.

The current through a DBS is given as

$$
I(V)=\frac{e}{\pi \hbar} \int d \epsilon T(\epsilon)\left[f_{l}(\epsilon)-f_{r}(\epsilon+e V)\right],
$$

where $f(\epsilon)$ is the Fermi-Dirac distribution function for the leads. As an example, the result for a $2 \mathrm{D}$ emitter model is presented in Fig. 4. The broadened shoulder in the high bias side of the main peak is due to plasmon assisted tunneling, though it is less distinct after averaging over the distribution function. Since we used an average charge density of $2 \times 10^{11} \mathrm{~cm}^{-2}$, the distance between the main peak and the shoulder is only a few $\mathrm{meV}$. Therefore this shoulder can easily disappear if the elastic coupling $\Gamma$ gets larger or other scattering effects become stronger.

To make direct comparison with the experiment, several effects need to be included. (i) The charge is dynamically stored in the well, i.e., $n$ itself is a function of the tunneling current (via $n=J / e \Gamma$ ). The $\omega_{q}$ also has a weak $\sqrt{n}$ dependence. Moreover, the coupling strength, $\left|g_{q}\right|^{2}$ has a much stronger density dependence $\left(\sim n^{3 / 2}\right)$. Therefore the intensity of the PAT current should decrease with the charge density in the well. (ii) The electron-electron interaction in the center well $\left(E_{e e}\right)$ shifts the resonant level, $\epsilon_{c} \rightarrow \epsilon_{c}^{\prime}=\epsilon_{c}+E_{e e}$. This, of course, is the cause of the current bistability. To obtain a qualitative picture, we have also included this dynamical charge feedback effect in our calculation selfconsistently. The $E_{e e}$ is modeled by a Hartree-Fock term [16] $E_{e e}=\alpha n$, where $\alpha$ is a form factor depending on the sample parameters. The result is plotted in Fig. 4. As expected, the inclusion of $E_{e e}$ is to tilt the resonant $I-V$ curve. Furthermore, due to the current-dependent electron-QWP coupling strength, the plasmon assisted tunneling current is reduced.

Finally, we would like to mention that a magnetic field applied in the $z$ direction should generally suppress the QWP due to the energy quantization in the $x-y$ plane which blocks the momentum relaxation. However, for a small B field, PAT can still occur as long as there is a finite overlap between the Landau levels. We recently observed such magnetic dependence of the PAT experimentally and the result is presented in Fig. 5. More detailed analysis of the magnetic field dependent result will be presented in a separate work.

In conclusion, the inelastic resonant tunneling due to electron-QWP coupling in a DBS is investigated. It is worth noting that plasmon structure in the electrical characteristics of DBSs is only likely to be observed in regions of apparent bistability since both effects accompany charge buildup in the well.

The authors would like to thank M. Henini for growing the sample. This work is supported in part by the Australian Research Council.

[1] R. Tsu and L. Esaki, Appl. Phys. Lett. 22, 562 (1973).

[2] T. C. L. G. Sollner et al., Appl. Phys. Lett. 43, 588 (1983).

[3] A. Zaslavski et al., Appl. Phys. Lett. 53, 1408 (1988); E. S. Alves et al., Electron. Lett. 24, 1190 (1988).

[4] T. C. L. G. Sollner, Phys. Rev. Lett. 59, 1622 (1987); V. J. Goldman, ibid. 59, 1623 (1987); T. J. Foster et al., Phys. Rev. B 39, 6205 (1989).

[5] M. L. Leadbeater et al., J. Phys. Condens. Matter 1, 10605 (1989).

[6] V. J. Goldman, D. C. Tsui, and J. E. Cunningham, Phys. Rev. B 36, 7635 (1987).

[7] A. D. Martin, M. L. F. Lerch, P. E. Simmonds, and L. Eaves, Appl. Phys. Lett. 64, 1248 (1994).

[8] M. L. F. Lerch, A. D. Martin, P. E. Simmonds, L. Eaves, and M. L. Leadbeater, Solid State Electron. 37, 961 (1994).

[9] M. L. Leadbeater et al., Semicond. Sci. Technol. 3, 1060 (1988); L. Eaves et al., Solid State Electron. 32, 1101 (1989).

[10] N. S. Wingreen, K. W. Jacobson, and J. Wilkins, Phys. Rev. Lett. 61, 1396 (1988); W. Cai et al., ibid. 63, 418 (1989).

[11] N. Zou and K. A. Chao, Phys. Rev. Lett. 69, 3224 (1992).

[12] G. D. Mahan, Many-Particle Physics (Plenum, New York, 1981).

[13] G. D. Mahan, Phys. Rev. 153, 882 (1967); D. L. Langreth, Phys. Rev. B 1, 471 (1970).

[14] J. Bardeen, Phys. Rev. Lett. 6, 57 (1961).

[15] P. M. Platzman and P. A. Wolff, Waves and Interactions in Solid State Plasmas (Academic, New York, 1973).

[16] D. D. Coon, K. Bandara, and H. Zhao, Appl. Phys. Lett. 54, 2115 (1989). 